\title{
Field-angle and DC-bias dependence of spin-torque diode in giant magnetoresistive microstripe
}

\author{
X. Li, ${ }^{1}$ Y. Zhou, ${ }^{2, a)}$ C. Zheng, ${ }^{1}$ P. H. Chan, ${ }^{1}$ M. Chan, ${ }^{3}$ and Philip W. T. Pong ${ }^{1, a)}$ \\ ${ }^{1}$ Department of Electrical and Electronic Engineering, The University of Hong Kong, Hong Kong, Hong Kong \\ ${ }^{2}$ School of Science and Engineering, Chinese University of Hong Kong (Shenzhen), Shenzhen 518172, China \\ ${ }^{3}$ Department of Electronic and Computer Engineering, Hong Kong University of Science and Technology, \\ Hong Kong, Hong Kong
}

(Received 24 August 2016; accepted 23 October 2016; published online 7 November 2016)

\begin{abstract}
The spin torque diode effect in all metal spintronic devices has been proposed as a microwave detector with a high power limit and resistivity to breakdown. The previous works have revealed the field-angle dependence of the rectified DC voltage $\left(V_{D C}\right)$ in the ferromagnetic stripe. The giant magnetoresistive (GMR) microstripe exhibits higher sensitivity compared with the ferromagnetic stripe. However, the influence of the magnetic field direction and bias current in the spin rectification of GMR microstripe is not yet reported. In this work, the angular dependence and bias dependence of resonant frequency $\left(f_{R}\right)$ and $V_{D C}$ are investigated. A macrospin model concerning the contribution of magnetic field, shape anisotropy, and unidirectional anisotropy is engaged to interpret the experimental data. $f_{R}$ exhibits a $\left|\sin \delta_{H}\right|$ dependence on the in-plane field angle $\left(\delta_{H}\right) . V_{D C}$ presents either $\left|\sin \delta_{H}\right|$ or $\left|\sin 2 \delta_{H} \cos \delta_{H}\right|$ relation, depending on the magnitude of $H_{\text {ext }}$. Optimized $V_{D C}$ of $24 \mu \mathrm{V}$ is achieved under $4 \mathrm{mT}$ magnetic field applied at $\delta_{H}=170^{\circ}$. Under out-of-plane magnetic field, $f_{R}$ shows a $\cos 2 \theta_{H}$ reliance on the polar angle $\left(\theta_{H}\right)$, whereas $V_{D C}$ is $\sin \theta_{H}$ dependent. The Oersted field of the DC bias current $\left(I_{D C}\right)$ modifies the effective field, resulting in shifted $f_{R}$. Enhanced $V_{D C}$ with increasing $I_{D C}$ is attributed to the elevated contribution of spin-transfer torque. Maximum $V_{D C}$ of $35.2 \mu \mathrm{V}$ is achieved, corresponding to $47 \%$ increase compared with the optimized value under zero bias. Higher $I_{D C}$ also results in enlarged damping parameter in the free layer, resulting in increased linewidth in the spin torque diode spectra. This work experimentally and analytically reveals the angular dependence of $f_{R}$ and $V_{D C}$ in the GMR microstripe. The results further demonstrate a highly tunable $f_{R}$ and optimized $V_{D C}$ by bias current without the external magnetic field. GMR microstripe holds promise for application as a high-power, frequency-tunable microwave detector that works under small or zero magnetic field. Published by AIP Publishing. [http://dx.doi.org/10.1063/1.4967175]
\end{abstract}

The emerging spintronic devices ${ }^{1}$ cover a broad spectrum of applications in microelectronics, ${ }^{2}$ energy engineering, ${ }^{3}$ and bioscience $^{4}$ through manipulating the interaction between the spin-transfer torque ${ }^{5}$ (STT) of electrons and the magnetization of magnetic materials. The previous observation of the radiofrequency (RF) oscillation excited by the spin-polarized current in a nanoscale spin valve has revealed the application as microwave oscillators. ${ }^{6,7}$ On the other hand, a DC voltage $\left(V_{D C}\right)$ is also generated when the RF current flows through the device as a result of the spin-torque diode effect. ${ }^{8}$ Over the past decade, intensive efforts have been devoted to developing spin-torque microwave detectors (STMDs) based on nanoscale magnetic tunnel junctions (MTJs). The recent demonstration of high microwave power detection sensitivity, over $12000 \mathrm{mV} / \mathrm{mW}$ and $75400 \mathrm{mV} / \mathrm{mW},{ }^{9,10}$ has proved the advantages of STMD, exceeding the current Schottky diode detectors. The application of MTJ-based STMD is limited in sensing relativity small signal due to the low microwave power limit and fragility to electrostatic shocks. ${ }^{11}$ The discovery of the spin rectification effect in ferromagnetic (FM) microstripe $^{12-21}$ has drawn a considerable attention as an approach to overcome the above limitations due to the current-in-plane (IP) geometry and the all-metal structure. Recently, it was

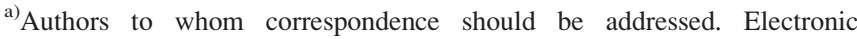
addresses: zhouyan@cuhk.edu.cn and ppong@eee.hku.hk.
}

shown that the giant magnetoresistive (GMR) microstripe exhibits more than ten times higher sensitivity compared with the FM microstripe. ${ }^{11,22,23}$ These investigations have revealed the influencing factors of $V_{D C}$ such as the stripe width ${ }^{23}$ and the interlayer coupling. ${ }^{11,24}$ Changing the magnitude and the direction of the in-plane (IP) and out-of-plane (OOP) magnetic fields was reported to be effective in tailoring $V_{D C}$ and at resonant frequency $\left(f_{R}\right)$ in MTJ-based STMD. ${ }^{25-27}$ The previous works focused on the spin rectification study of the GMR microstripe under the IP magnetic field. ${ }^{22,24,28}$ However, the OOP-field-angle dependence of $f_{R}$ and $V_{D C}$ remains unclear. In addition, DC bias was reported as a powerful method to improve the sensitivity of MTJ-based STMD. ${ }^{29,30}$ The GMR stripe can bear the large DC current in the order of tens of $\mathrm{mA}$, which offers a broad potential for further enhancing the sensitivity. In this work, the microwave rectification performance of GMR microstripe was first investigated at zero bias under the changing magnitude and the direction of the IP and OOP magnetic fields. Later, the DC bias current was applied to evaluate its modulation capability on $V_{D C}$ and $f_{R}$. The investigations of the approaches to optimize $V_{D C}$ and tune $f_{R}$ would boost the development of STMD based on the GMR stripe.

The GMR multilayers of Ta 3.5/Cu 15/IrMn 5.5/CoFe 4/ $\mathrm{Ru}$ 0.7/CoFe 3/Cu 6.5/NiFe 25/Ta 3.5 (thickness in nanometer) were prepared on the thermally oxidized silicon wafer. The thickness of the $\mathrm{Cu}$ spacer was chosen to be $6.5 \mathrm{~nm}$ for an 
optimized MR ratio and reduced coupling between the pinned layer and the free layer. ${ }^{31}$ Electron beam lithography and Argon ion milling were engaged to pattern the $2.5 \mu \mathrm{m} \times 20 \mu \mathrm{m}$ microstripes. The optical microscopic image of the sample after $\mathrm{Cu}$ contact pads deposition is shown in Fig. 1(a). The direction of the magnetization of the reference layer $(\mathbf{M}$, $x$-axis) was designed to be normal to the longitudinal axis of the stripe (y-axis) to enhance the spin rectification effect. ${ }^{23}$ During the experiment, the external magnetic field $\left(H_{\text {ext }}\right)$ was applied with the polar angle $\left(\theta_{H}\right)$ and azimuthal angle $\left(\delta_{H}\right)$ (Fig. 1(b)). Due to the strong demagnetization field, the magnetization of the free layer $(\mathbf{m})$ is rotated within the $x-y$ plane at an azimuthal angle $\left(\delta_{F}\right)$. The measured MR ratio at the $x$-direction is $1.1 \%$ (Fig. 1(c)). In the microwave rectification characterization (Fig. 1(d)), the pulse-modulated microwave current and the DC bias current were mixed through a biasTee and injected into the microstripe. The rectified voltage $\left(V_{\text {mix }}\right)$ was measured by a lock-in amplifier, whereas the microwave frequency swept from $0.1 \mathrm{GHz}$ to $6 \mathrm{GHz}$. The cable loss is estimated to be $3 \mathrm{~dB}$ at $2 \mathrm{GHz}$. The output power of the microwave synthesizer is flattened over the frequency range to compensate for the frequency-dependent losses.

When the current flows along the stripe, a significant portion of the current is shunt by a $15-\mathrm{nm}$-thick $\mathrm{Cu}$ bottom electrode $(64.4 \%)$ and a 6.5 -nm-thick $\mathrm{Cu}$ spacer $(23.6 \%)$. The two $\mathrm{Cu}$ layers also contribute to the majority of the Oersted field on the NiFe free layer. Assuming the current flows homogenously in each layer, the Oersted field contributed by one layer can be estimated through integration within the cross-section using Ampère's circuital law. The Oersted
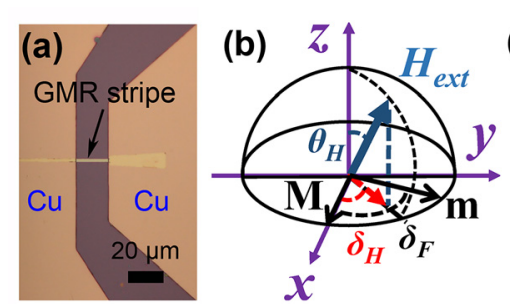

(d)
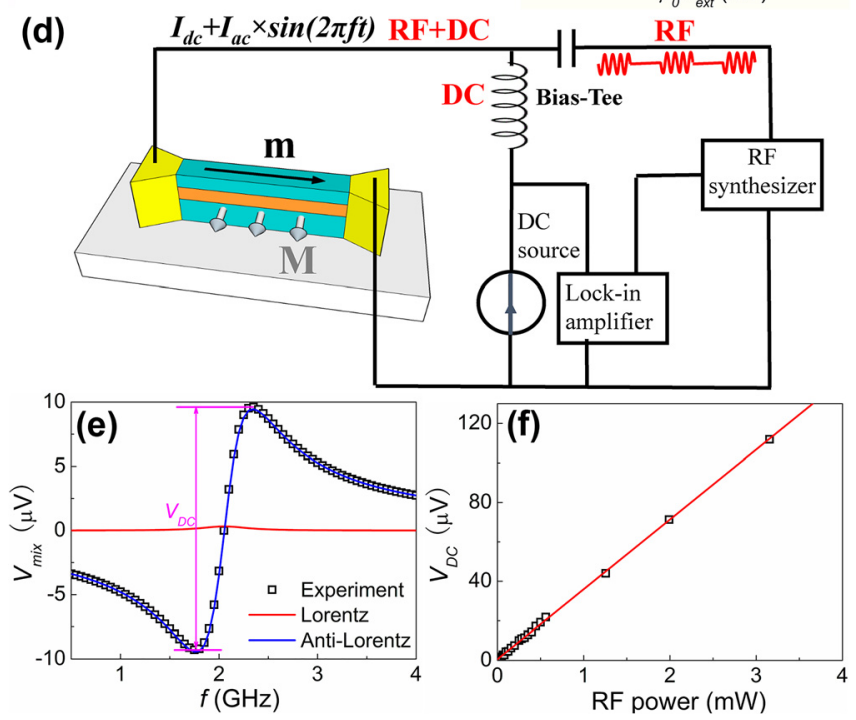

FIG. 1. (a) The optical microscopic image of the microstripe, (b) schematic of the axis system, (c) the $x$-direction MR curve, (d) the experimental setup for measuring the spin-torque diode spectrum, (e) the typical $V_{m i x}-f$ spectra, and (f) $V_{D C}$ as a function of the microwave power. field in the free layer is calculated as the summation of the contribution from the current distributed in each layer. When $2.4 \mathrm{~mA}$ microwave current $(-3 \mathrm{dBm})$ is applied, the amplitude of the alternating Oersted field is $0.53 \mathrm{mT}$. The magnetization dynamics of the free layer is thus stimulated by the Oersted field $^{22,23}$ and the spin-transfer torque ${ }^{12}$ of the microwave current, resulting in the coexistence of a symmetric Lorentz part and an asymmetric anti-Lorentz component in the $V_{\text {mix }}-f$ spectrum $^{32,33}$

$$
\begin{aligned}
V_{\mathrm{mi} x}= & A I_{R F}^{2}+I_{R F}^{2}\left[B \frac{1}{1+\left(\left(f-f_{R}\right) / \sigma\right)^{2}}\right. \\
& \left.-C \frac{f-f_{R}}{\sigma} \frac{1}{1+\left(\left(f-f_{R}\right) / \sigma\right)^{2}}\right],
\end{aligned}
$$

where $\sigma$ is the linewidth, $\mathrm{A}$ is the non-resonant background, and $\mathrm{B}$ and $\mathrm{C}$ are proportional to the amplitude of the symmetric and antisymmetric components, respectively. The $f_{R}$ and $\sigma$ are acquired through fitting the $V_{m i x}-f$ spectrum with Eq. (1), as shown in Fig. 1(e). The high content of the asymmetric component indicates the dominating role of the spin rectification effect contributed by the field torque. $V_{D C}$ is calculated as the voltage difference between the peak and trough in the $V_{\text {mix }}-f$ spectrum. As microwave power increases from $1.6 \mu \mathrm{W}$ to $3.15 \mathrm{~mW}, V_{D C}$ increases linearly from $0.06 \mu \mathrm{V}$ to $112 \mu \mathrm{V}$ (Fig. 1(f)). The broad operation-power range proves the eligibility of GMR-stripe as a high power microwave detector. In the following investigations, the microwave power is fixed at $-3 \mathrm{dBm}$.

The spin-torque diode effect was first investigated under the IP magnetic field $\left(\theta_{H}=90^{\circ}, \delta_{H}=0^{\circ}-360^{\circ}\right)$. When $\delta_{H}=0^{\circ}, f_{R}$ exhibits a v-shape reliance on $H_{\text {ext }}$ (Fig. 2(a)). The larger $f_{R}$ under the positive field as compared to $f_{R}$ under the negative field $(3.13 \mathrm{GHz}$ at $30 \mathrm{mT}$ compared with $3.01 \mathrm{GHz}$ at $-30 \mathrm{mT}$ ) indicates that the pinned layer has introduced a unidirectional anisotropy $\left(H_{U A}\right)$ in the free layer along the $+x$ direction through orange peel coupling. ${ }^{34}$ An analytical model $^{26,27,35}$ is proposed considering the competing effect of $H_{e x t}, H_{U A}$, and the shape anisotropy $\left(H_{S A}\right){ }^{29,30,38}$ The IP shift angle of $\mathbf{m}\left(\delta_{F}\right)$ can be inferred from the following equation: ${ }^{36}$

$$
H_{e x t} \sin \theta_{H} \sin \left(\delta_{F}-\delta_{H}\right)+H_{U A} \sin \delta_{F}=\frac{1}{2} H_{S A} \sin 2 \delta_{F} .
$$

According to Kittel's ferromagnetic resonance model ${ }^{37,38}$

$$
f_{R}=\frac{\gamma}{2 \pi} \sqrt{H_{e f f}\left(H_{e f f}+4 \pi m_{S}\right)}
$$

where $\gamma=1.76 \times 10^{7} \mathrm{~Hz} / \mathrm{Oe}$ is the gyromagnetic ratio of electrons and $\mathrm{m}_{\mathrm{S}}=7.6 \times 10^{5} \mathrm{~A} / \mathrm{m}$ is the saturation magnetization of the NiFe free layer. $H_{\text {eff }}$ refers to the IP effective field calculated from the following equation:

$$
H_{e f f}=\left|H_{e x t} \cos \left(\delta_{F}-\delta_{H}\right)-H_{S A} \cos 2 \delta_{F}+H_{U D} \cos \delta_{F}\right| .
$$

In a preliminary qualitative investigation, the simple case of $H_{U A}=0$ is considered. When $\theta_{H}=90^{\circ}$ and $\delta_{H}=0^{\circ}, H_{\text {eff }}$ can be expressed as $H_{e f f}=\left|-H_{e x t}^{2} / H_{S A}+H_{S A}\right|$. As a result, $H_{\text {eff }}$ and $f_{R}$ decrease with increasing $H_{\text {ext }}$ when $H_{e x t}<H_{S A}$, and then 
increase with $H_{\text {ext }}$ afterward. Similar $f_{R}-H_{e x t}$ relation under the hard-axis magnetic field has also been reported in the MTJbased STMD. ${ }^{26,39}$ The $f_{R}-H_{\text {ext }}$ relation when $\delta_{H}=30^{\circ}, 60^{\circ}$, and $90^{\circ}$ is further quantitatively simulated based on Eq. (3). The parameters of $\mu_{0} H_{S A}=7.6 \mathrm{mT}$ and $\mu_{0} H_{U A}=1 \mathrm{mT}$ are extrapolated from the fit. $V_{D C}$ is plotted against $H_{e x t}$ in Fig. 2(b). Higher $V_{D C}$ is achieved at relatively smaller $H_{\text {ext }}$, since the angle $(\varphi)$ between $\mathbf{m}$ and $\mathbf{M}$ approaches $90^{\circ}$ when $H_{\text {ext }}$ is near zero, and $V_{D C}$ is proportional to $(\sin \varphi)^{2}{ }^{22}$ When $\delta_{F}=0^{\circ}$, maximum $V_{D C}$ is achieved at $\mu_{0} H_{e x t}=\sim-2 \mathrm{mT}$. This is also an evidence for the positive $H_{U A}$, as the negative field is required to overcome $H_{U A}$ to maintain the perpendicular alignment between $\mathbf{m}$ and $\mathbf{M}$. When $\delta_{H}=90^{\circ}, V_{D C}$ reaches the maximum under zero magnetic field. The IP angular dependence of $f_{R}$ is shown in Fig. 2(c). The smaller the $f_{R}$ at $\delta_{H}=180^{\circ}$ compared with $\delta_{H}=0^{\circ}$ is attributed to the $H_{U A}$ at the $+x$ direction. The $f_{R^{-}} \delta_{H}$ relation is consistent with the previous experimental report on the angular dependence of $f_{R}$ in the NiFe stripe. ${ }^{15,17}$ When a $\cos 2 \delta_{H}$ fitting was previously used, the experimental observations in these Refs. 19 and 21 suggest it is more likely a $\left|\sin \delta_{H}\right|$ relation, as proved by our experimental and simulation results. The IP angular dependence of $V_{D C}$ when $\mu_{0} H_{\text {ext }}=4 \mathrm{mT}$, on the other hand, exhibits a deformed $\left|\sin \delta_{H}\right|$ relation (Fig. 2(d)) due to $H_{U A}$. This result is qualitatively similar to the calculated $(\sin \varphi)^{2}-\delta_{H}$ relation shown in the inset of Fig. 2(d), as $V_{D C}$ is proportional to $(\sin \varphi)^{2}{ }^{22}$ This $V_{D C}-\delta_{H}$ relation is consistent with the previous report in the $\mathrm{NiFe}$ stripe that $V_{D C} \propto\left|\sin \delta_{H}\right|$ when $H_{\text {ext }} \ll H_{S A} \cdot{ }^{15}$ In contrast, $V_{D C}$ exhibits a $\left|\sin 2 \delta_{H} \cos \delta_{H}\right|$ relation when $\mu_{0} H_{\text {ext }}=15 \mathrm{mT}$, which is also consistent with the previous report when $H_{e x t} \gg H_{S A}{ }^{15}$ These results show that a wide frequency range (from $1.9 \mathrm{GHz}$ to $3 \mathrm{GHz}$ ) and optimized $V_{D C}$ (up to $24 \mu \mathrm{V}$ ) can be achieved through manipulating the IP angle of the $4 \mathrm{mT}$ magnetic field.

The OOP angular dependence was investigated through changing the tilt angle of hard-axis magnetic field $\left(\theta_{H}=0^{\circ}-360^{\circ}, \delta_{H}=0^{\circ}\right)$. When $\mu_{0} H_{\text {ext }}$ is increased from 0 to $30 \mathrm{mT}$, a similar v-shape $f_{R^{-}} H_{\text {ext }}$ relation is observed (Fig. 3(a)). When $H_{\text {ext }}$ is perpendicular to the plane $\left(\theta_{H}=0^{\circ}\right), f_{R}$ is nearly independent on $H_{\text {ext }}$. This is because $f_{R}$ is dominated by the in-plane $H_{\text {eff }}$ since $\mathbf{m}$ is constrained in-plane by the large
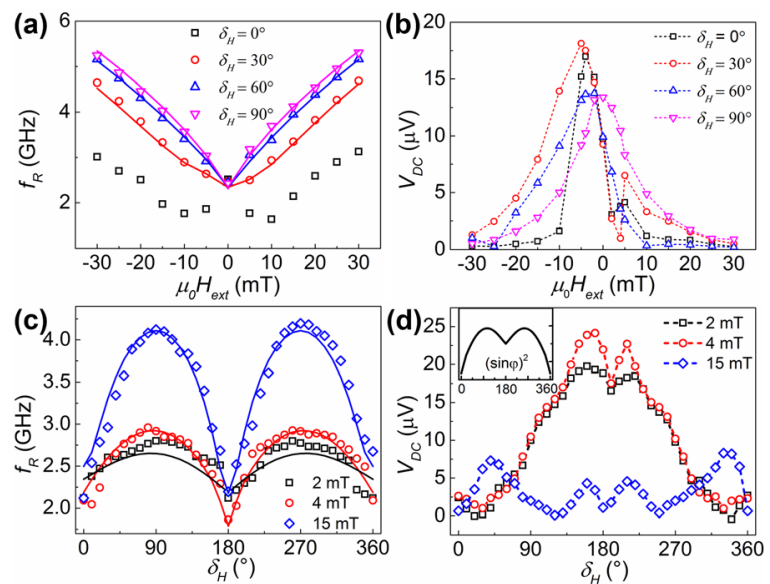

FIG. 2. The influence of the IP magnetic field: (a) $f_{R}$ and (b) $V_{D C}$ as a function of $H_{e x t}$; (c) $f_{R}$ and (d) $V_{D C}$ and as a function of $\delta_{H}$ (inset: the simulated $(\sin \varphi)^{2}-\delta_{H}$ relation when $\left.\mu_{0} H_{\text {ext }}=2 \mathrm{mT}\right)$. The scatters refer to the experimental data, whereas the solid lines indicate the numerical simulation. demagnetization in the $z$-direction, whereas in-plane $H_{\text {eff }}$ is not influenced by a perpendicular $H_{\text {ext }}$. Similarly, $V_{D C}$ is also expected to be independent on $H_{\text {ext }}$ when $\theta_{H}=0^{\circ}$, since $\varphi$ is not affected by $H_{\text {ext }}$. The drift of $V_{D C}$ with $H_{\text {ext }}$ shown in Fig. 3 (b) is due to the offset of field angle from $0^{\circ}$. The discrepancy between the experimental data and the numerical simulation is attributed to the multi-domain geometry in the stripe, which deviates from the single domain approach in simulation. $H_{U A}$ is also responsible for the shift of the peak of $V_{D C}$ to the negative field (Fig. 3(b)). The OOP angular dependence of $f_{R}$ and $V_{D C}$ are shown in Figs. 3(c) and 3(d), respectively. $f_{R}$ exhibits an asymmetric $\cos \left(2 \theta_{H}\right)$ reliance on the polar angle of $H_{\text {ext }}$, as confirmed by the numerical simulation in the solid lines in Fig. 3(c). This angular dependence is also qualitatively resolved by the model in Eq. (3) without considering $H_{U A}$. Assuming that $H_{\text {ext }} \sin \theta_{H} \ll H_{S A} \ll 4 \pi m_{S}, f_{R}$ can be expressed as

$$
\begin{aligned}
f_{R} & =\frac{\gamma}{2 \pi} \sqrt{4 \pi m_{S}\left(-\frac{H_{e x t}^{2} \sin ^{2} \theta_{H}}{H_{S A}}+H_{S A}\right)} \\
& \approx \frac{\gamma}{2 \pi} \sqrt{4 \pi m_{S}}\left(\sqrt{H_{S A}}-\frac{H_{e x t}^{2}}{2\left(H_{S A}\right)^{3 / 2}} \sin ^{2} \theta_{H}\right) \propto \cos 2 \theta_{H} .
\end{aligned}
$$

On the other hand, $V_{D C}$ exhibits a sinusoidal dependence on $\theta_{H}$. This angular dependence can also be explicated analytically without considering $H_{U A}$. The microwave field $\left(H_{R F} \times \sin (2 \pi f t)\right)$ stimulates the steady-state oscillation in $\mathbf{m}$ with a small angle of $\Delta \delta_{F}$. It can be inferred from Eq. (2) that $\cos \delta_{F}=H_{e x t} \sin \theta_{H} / H_{S A}$ and $\Delta \delta_{F} \approx H_{R F} /\left(H_{S A} \sin \delta_{F}\right)$. In a GMR multilayer, the resistance is a function of $\varphi$ $\left(R=2 R_{A P} R_{P} /\left[\left(R_{A P}+R_{P}\right)+\left(R_{A P}-R_{P}\right) \cdot \cos \varphi\right]\right.$, where $R_{A P}$ and $R_{P}$ are the resistances when $\mathbf{m}$ and $\mathbf{M}$ are antiparallel or parallel, respectively). ${ }^{10,32,40,41}$ Considering $\varphi=\delta_{F}$ and $\left(R_{A P}-R_{P}\right) / R_{P}=0.011 \ll 1$, the resistance change due to the oscillation of $\mathbf{m}$ can be estimated as

$$
\begin{aligned}
\Delta R & =\frac{d R}{d \delta_{F}} \Delta \delta_{F}=\frac{R_{P}}{2\left(1+\frac{M R}{2} \cos \delta_{F}\right)^{2}} \frac{M R \times H_{R F}}{H_{S A}} \\
& \approx \frac{M R \times H_{R F} R_{P}}{2 H_{S A}}\left(1-M R \cos \delta_{F}\right) .
\end{aligned}
$$

$V_{D C}$ is calculated from ${ }^{42}$

$$
\begin{aligned}
V_{D C} & \propto \Delta R \times I_{R F}=\frac{M R \times H_{R F} R_{P} I_{R F}}{2 H_{S A}}\left(1-M R \cos \delta_{F}\right) \\
& \propto\left(1-M R \frac{H_{e x t} \sin \theta_{H}}{H_{S A}}\right) \propto-\sin \theta_{H} .
\end{aligned}
$$

These results demonstrate that $f_{R}$ increases from $1.95 \mathrm{GHz}$ to $2.56 \mathrm{GHz}$ and a maximum $V_{D C}$ of $20.36 \mu \mathrm{V}$ is obtained through adjusting $\theta_{H}$ of $4 \mathrm{mT}$ hard axis field.

In order to explore the approaches to improve $V_{D C}$ of the GMR stripe, DC current $\left(I_{D C}\right)$ is applied during the experiments. The $V_{\text {mix }}-f$ spectra under zero field and $2 \mathrm{mT}$ field at the $+x$-direction are plotted in Fig. 4(a). As $I_{D C}$ changes from $-15 \mathrm{~mA}$ to $15 \mathrm{~mA}, f_{R}$ first increases as the negative $I_{D C}$ is 

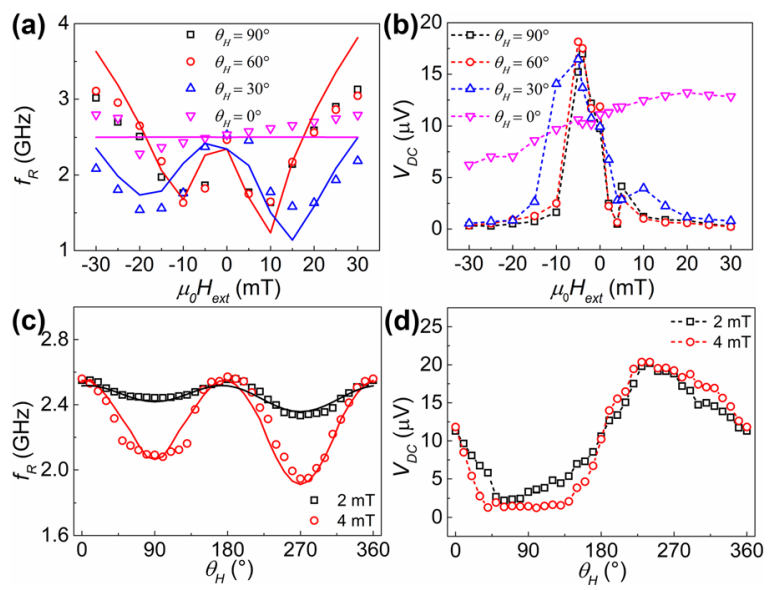

FIG. 3. The performance under $x$-z plane magnetic field: (a) $f_{R}$ and (b) $V_{D C}$ as a function of $H_{e x t}$, (c) $f_{R}$ and (d) $V_{D C}$ and as a function of $\theta_{H}$. The scatters refer to experimental data, whereas the solid lines indicate the numerical simulation.

reduced, and then decreases as $I_{D C}$ turns to be positive (Fig. 4(b)). This parabolic-shape $f_{R}-I_{D C}$ relation is attributed to the modulation of the Oersted field of the DC bias current. When $I_{D C}=15 \mathrm{~mA}$, the Oersted DC field is estimated to be $3.4 \mathrm{mT}$. The Oersted field has resulted in the shift in $f_{R}$ to $2 \mathrm{GHz}$ when $\mu_{0} H_{\text {ext }}=0 \mathrm{mT}$, similar to the value under $3.4 \mathrm{mT}$ external field extrapolated from the $f_{R}-H_{\text {ext }}$ relation in Fig. 3(a). As $I_{D C}$ is increased from $0 \mathrm{~mA}$ to $\pm 13 \mathrm{~mA}$, a gradual enhancement in $V_{D C}$ is observed (Fig. 4(c)). The IP spin-transfer torque is reported to increase with the external bias voltage in MTJ. ${ }^{43,44}$ The higher $V_{D C}$ is attributed to the increased contribution of spin-transfer torque, as evidenced by the enhanced symmetry in the $V_{m i x}-f$ peak. As $I_{D C}$ is further increased, $V_{D C}$ tends to decrease. This is explained by the increased influence of the Oersted field, since $V_{D C}$ drastically decreases when $\mu_{0} H_{\text {ext }}$ is beyond the range of $-2 \mathrm{mT}$ to $-4 \mathrm{mT}$, as shown in Fig. 3(a). The maximum $V_{D C}$ of $35.2 \mu \mathrm{V}$, acquired when $\mu_{0} H_{\text {ext }}=2 \mathrm{mT}$ and $I_{D C}=-13 \mathrm{~mA}$, is 16 times as large as when $I_{D C}=0 \mathrm{~mA}$. This value is also much higher than the optimized $V_{D C}$ under tilted IP (cf. $24 \mu \mathrm{V}$ ) or OOP (cf. $20.4 \mu \mathrm{V}$ ) magnetic fields under zero bias. $\sigma$ is also increased under higher DC bias, as shown in Fig. 4(d). When $\mu_{0} H_{\text {ext }}=0 \mathrm{mT}, \sigma$ is determined by the following equation ${ }^{45}$

$$
\sigma=\frac{\alpha(-\gamma)}{2 \pi}\left(4 \pi m_{S}+H_{S A}\right),
$$

where $\alpha$ is the Gilbert damping factor. As $I_{D C}$ increases from $0 \mathrm{~mA}$ to $-13 \mathrm{~mA}, \alpha$ is increased from 0.009 to 0.013 . The increase in $\sigma$ is believed to arise from the increased $\alpha$ due to the DC bias, similar to the reports in the MTJ-based spin torque diode. $^{30,46}$

The above results have demonstrated an improved $V_{D C}$ through tailoring the magnetic field angle and applying the DC bias current. Although the optimized microwave detection sensitivity is still much lower than the TMR-based STMD, the GMR stripe still offers some advantages. First, the fabrication of GMR microstripe only requires one or two steps of patterning and etching, whereas the MTJ nanopillar requires multisteps of lithography and deposition to define the current-perpendicular-to-plane structure. Second, the resistance of GMR stripe can be easily modified through tailoring the stripe width or length to match the characteristic impedance of the RF

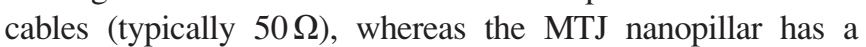
much higher resistance of several hundred or thousand Ohms. Third, the GMR stripe exhibits a linear response to microwave current over $6 \mathrm{~mA}$, which is much larger than the several hundred microampere in the previously reported MTJ-based STMDs. ${ }^{47}$ Finally, when the operation under zero magnetic field was also reported in some MTJ-based STMD, ${ }^{9}$ the GMR stripe further exhibits tuned $f_{R}$ by the DC bias current, which enables the operation over a wider frequency range without the need of magnetic field tuning $(1.8 \mathrm{GHz}-2.6 \mathrm{GHz}$ under zero field as $I_{D C}$ changes from $-15 \mathrm{~mA}$ to $15 \mathrm{~mA}$ ). Moreover, the sensitivity of GMR-stripe-based STMD can be further increased through engaging the state-of-art GMR technology (MR ratio as high as $74.8 \%$ in Heusler-alloys-based GMR). ${ }^{48}$ These efforts will enhance the eligibility of GMR-stripe as a potential low-cost, high-power, and frequency-tunable microwave detector.

In summary, the spin torque diode effect in the GMR microstripe was investigated through modifying the tilt angle of the IP and OOP magnetic fields and the DC bias current. A model considering the influence of the shape anisotropy and unidirectional anisotropy is proposed to quantitatively and analytically resolve the experimental data. When the IP magnetic field is applied, $f_{R}$ shows $\left|\sin \delta_{H}\right|$ dependence on the field angle, as $H_{\text {eff }}$ is modified by the competition between anisotropy and the magnetic field. $V_{D C}$ is $\left|\sin \delta_{H}\right|$ dependent when $H_{\text {ext }} \ll H_{S A}$, or $\left|\sin 2 \delta_{H} \cos \delta_{H}\right|$ is dependent when $H_{\text {ext }} \gg$ $H_{S A}$. Under OOP magnetic field, $f_{R}$ presents $\cos 2 \delta_{H}$ reliance on the polar angle of $H_{\text {ext }}$, whereas $f_{R}$ is sinusoidally dependent, since $H_{e x t}$ is far below the demagnetization field. The reduced $f_{R}$ with increasing $I_{D C}$ is attributed to the modified $H_{\text {ext }}$ due to the Oersted field from the DC current. The maximum $V_{D C}$ of $35.2 \mu \mathrm{V}$ is achieved under $I_{D C}=-13 \mathrm{~mA}$ due to the increased contribution of the spin-transfer torque. The higher $I_{D C}$ also results in increased $\alpha$, which is responsible for the increase in $\sigma$. This work has revealed the field-angle and bias dependence of the spin-rectification effect in GMR microstripes. The results gain insight on the sensitivity optimization and frequency modulation in GMR microstripes.
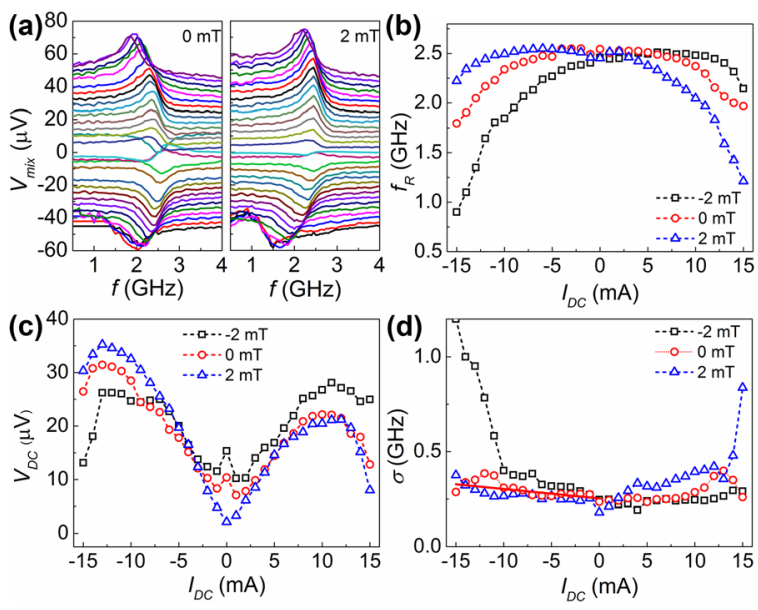

FIG. 4. The bias dependence of spin rectification under the $x$-direction magnetic field: (a) Spin-torque diode spectra measured when $\mu_{0} H_{e x t}=0$ and $2 \mathrm{mT}$ ( $I_{D C}$ changes from $-15 \mathrm{~mA}$ to $15 \mathrm{~mA}$ from top to bottom, each spectrum is shifted by $3 \mu \mathrm{V}$ ), (b) $f_{R}$, (c) $V_{D C}$, and (d) $\sigma$ as a function of $I_{D C}$. 
This research was supported by the Seed Funding Program for Basic Research, Seed Funding Program for Applied Research, and Small Project Funding Program from the University of Hong Kong, ITF Tier 3 funding (ITS/203/ 14, ITS/104/13, ITS/214/14), RGC-GRF grant (HKU 17210014), Innovation and Technology Fund Internship Programme (InP/182/14), and University Grants Committee of Hong Kong (AoE/P-04/08). Y.Z. also acknowledges the support by the National Natural Science Foundation of China (Project No. 1157040329) and Shenzhen Fundamental Research Fund under Grant No. JCYJ20160331164412545.

${ }^{1}$ S. D. Bader and S. S. P. Parkin, Annu. Rev. Condens. Matter Phys. 1, 71 (2010). ${ }^{2}$ S. A. Wolf, D. D. Awschalom, R. A. Buhrman, J. M. Daughton, S. von Molnár, M. L. Roukes, A. Y. Chtchelkanova, and D. M. Treger, Science 294, 1488 (2001).

${ }^{3}$ S. Hemour, Y. Zhao, C. H. P. Lorenz, D. Houssameddine, Y. Gui, C.-M. Hu, and K. Wu, IEEE Trans. Microwave Theory Technol. 62, 965 (2014).

${ }^{4}$ L. P. Ichkitidze, N. A. Bazaev, D. V. Telyshev, R. Y. Preobrazhensky, and M. L. Gavrushina, Biomed. Eng. 48, 305 (2015).

${ }^{5}$ E. B. Myers, D. C. Ralph, J. A. Katine, R. N. Louie, and R. A. Buhrman, Science 285, 867 (1999).

${ }^{6}$ S. I. Kiselev, J. C. Sankey, I. N. Krivorotov, N. C. Emley, R. J. Schoelkopf, R. A. Buhrman, and D. C. Ralph, Nature 425, 380 (2003).

${ }^{7}$ W. H. Rippard, M. R. Pufall, S. Kaka, S. E. Russek, and T. J. Silva, Phys. Rev. Lett. 92, 027201 (2004).

${ }^{8}$ A. A. Tulapurkar, Y. Suzuki, A. Fukushima, H. Kubota, H. Maehara, K. Tsunekawa, D. D. Djayaprawira, N. Watanabe, and S. Yuasa, Nature 438, 339 (2005).

${ }^{9}$ B. Fang, M. Carpentieri, X. Hao, H. Jiang, J. A. Katine, I. N. Krivorotov, B. Ocker, J. Langer, K. L. Wang, B. Zhang, B. Azzerboni, P. K. Amiri, G. Finocchio, and Z. Zeng, Nature Commun. 7, 11259 (2016).

${ }^{10}$ S. Miwa, S. Ishibashi, H. Tomita, T. Nozaki, E. Tamura, K. Ando, N. Mizuochi, T. Saruya, H. Kubota, K. Yakushiji, T. Taniguchi, H. Imamura, A. Fukushima, S. Yuasa, and Y. Suzuki, Nature Mater. 13, 50 (2014).

${ }^{11}$ S. Ziętek, P. Ogrodnik, W. Skowroński, P. Wiśniowski, M. Czapkiewicz, T. Stobiecki, and J. Barnaś, Appl. Phys. Lett. 107, 122410 (2015).

${ }^{12}$ A. Yamaguchi, H. Miyajima, T. Ono, Y. Suzuki, S. Yuasa, A. Tulapurkar, and Y. Nakatani, Appl. Phys. Lett. 90, 182507 (2007).

${ }^{13}$ N. Mecking, Y. S. Gui, and C. M. Hu, Phys. Rev. B 76, 224430 (2007).

${ }^{14}$ A. Thiaville and Y. Nakatani, J. Appl. Phys. 104, 093701 (2008).

${ }^{15}$ A. Yamaguchi, K. Motoi, A. Hirohata, H. Miyajima, Y. Miyashita, and Y. Sanada, Phys. Rev. B 78, 104401 (2008).

${ }^{16}$ A. Yamaguchi, K. Motoi, A. Hirohata, and H. Miyajima, Phys. Rev. B 79, 224409 (2009).

${ }^{17}$ A. Yamaguchi, K. Motoi, H. Miyajima, and Y. Nakatani, J. Appl. Phys. 105, $07 \mathrm{D} 301$ (2009).

${ }^{18}$ O. Mosendz, J. E. Pearson, F. Y. Fradin, G. E. W. Bauer, S. D. Bader, and A. Hoffmann, Phys. Rev. Lett. 104, 046601 (2010).

${ }^{19}$ X. Fan, W. Wang, Y. Wang, H. Zhou, J. Rao, X. Zhao, C. Gao, Y. S. Gui, C.-M. Hu, and D. Xue, Appl. Phys. Lett. 105, 262404 (2014).

${ }^{20}$ A. Ganguly, K. Kondou, H. Sukegawa, S. Mitani, S. Kasai, Y. Niimi, Y. Otani, and A. Barman, Appl. Phys. Lett. 104, 072405 (2014).
${ }^{21}$ Y. Zhang, X. Fan, X. Zhao, J. Rao, H. Zhou, D. Guo, Y. S. Gui, C.-M. Hu, and D. Xue, J. Appl. Phys. 117, 023905 (2015).

${ }^{22}$ S. Ziętek, P. Ogrodnik, M. Frankowski, J. Chęciński, P. Wiśniowski, W. Skowroński, J. Wrona, T. Stobiecki, A. Żywczak, and J. Barnaś, Phys. Rev. B 91, 014430 (2015).

${ }^{23}$ J. Kleinlein, B. Ocker, and G. Schmidt, Appl. Phys. Lett. 104, 153507 (2014).

${ }^{24}$ A. A. Timopheev, N. A. Sobolev, Y. G. Pogorelov, S. A. Bunyaev, J. M. Teixeira, S. Cardoso, P. P. Freitas, and G. N. Kakazei, J. Appl. Phys. 113, 17D713 (2013).

${ }^{25}$ C. Wang, Y. T. Cui, J. Z. Sun, J. A. Katine, R. A. Buhrman, and D. C. Ralph, Phys. Rev. B 79, 224416 (2009).

${ }^{26}$ X. Li, C. Zheng, Y. Zhou, H. Kubota, S. Yuasa, and P. W. T. Pong, Appl. Phys. Lett. 108, 232407 (2016).

${ }^{27}$ T. Taniguchi and H. Imamura, J. Appl. Phys. 114, 053903 (2013).

${ }^{28}$ T. E. P. Bueno, D. E. Parreiras, G. F. M. Gomes, S. Michea, R. L. Rodríguez-Suárez, M. S. A. Filho, W. A. A. Macedo, K. Krambrock, and R. Paniago, Appl. Phys. Lett. 104, 242404 (2014).

${ }^{29}$ Y. Shiota, S. Miwa, S. Tamaru, T. Nozaki, H. Kubota, A. Fukushima, Y. Suzuki, and S. Yuasa, Appl. Phys. Lett. 105, 192408 (2014).

${ }^{30}$ S. Ishibashi, K. Ando, T. Seki, T. Nozaki, H. Kubota, S. Yakata, H. Maehara, A. Fukushima, S. Yuasa, and Y. Suzuki, IEEE Trans. Magn. 47, 3373 (2011)

${ }^{31}$ T. G. S. M. Rijks, R. Coehoorn, J. T. F. Daemen, and W. J. M. de Jonge, J. Appl. Phys. 76, 1092 (1994).

${ }^{32}$ M. Harder, Y. Gui, and C.-M. Hu, preprint arXiv:1605.00710 (2016).

${ }^{33}$ J. Zhu, J. A. Katine, G. E. Rowlands, Y.-J. Chen, Z. Duan, J. G. Alzate, P. Upadhyaya, J. Langer, P. K. Amiri, K. L. Wang, and I. N. Krivorotov, Phys. Rev. Lett. 108, 197203 (2012).

${ }^{34}$ W. F. Egelhoff, P. J. Chen, C. J. Powell, M. D. Stiles, and R. D. McMichael, J. Appl. Phys. 79, 2491 (1996).

${ }^{35}$ R. Matsumoto, H. Kubota, T. Yamaji, H. Arai, S. Yuasa, and H. Imamura, Jpn. J. Appl. Phys., Part 1 53, 123001 (2014).

${ }^{36}$ R. Matsumoto, A. Chanthbouala, J. Grollier, V. Cros, A. Fert, K. Nishimura, Y. Nagamine, H. Maehara, K. Tsunekawa, A. Fukushima, and S. Yuasa, Appl. Phys. Express 4, 063001 (2011).

${ }^{37}$ C. Kittel, Phys. Rev. 71, 270 (1947).

${ }^{38}$ C. Kittel, Phys. Rev. 73, 155 (1948).

${ }^{39}$ Z. Zeng, K. H. Cheung, H. W. Jiang, I. N. Krivorotov, J. A. Katine, V. Tiberkevich, and A. Slavin, Phys. Rev. B 82, 100410 (2010).

${ }^{40}$ Y. Zhou, S. Bonetti, J. Persson, and J. Akerman, IEEE Trans. Magn. 45, $2421(2009)$.

${ }^{41}$ Y. Zhou, J. Persson, and J. Akerman, J. Appl. Phys. 101, 09A510 (2007).

${ }^{42}$ J. C. Sankey, P. M. Braganca, A. G. F. Garcia, I. N. Krivorotov, R. A. Buhrman, and D. C. Ralph, Phys. Rev. Lett. 96, 227601 (2006).

${ }^{43}$ W. Skowronski, M. Frankowski, J. Wrona, T. Stobiecki, P. Ogrodnik, and J. Barnas, Appl. Phys. Lett. 105, 072409 (2014).

${ }^{44}$ H. Kubota, A. Fukushima, K. Yakushiji, T. Nagahama, S. Yuasa, K. Ando, H. Maehara, Y. Nagamine, K. Tsunekawa, D. D. Djayaprawira, N. Watanabe, and Y. Suzuki, Nat. Phys. 4, 37 (2008).

${ }^{45}$ B. K. Kuanr, R. E. Camley, and Z. Celinski, J. Magn. Magn. Mater. 286, 276 (2005).

${ }^{46}$ Y. Suzuki and H. Kubota, J. Phys. Soc. Jpn. 77, 031002 (2008).

${ }^{47}$ Y. S. Gui, Y. Xiao, L. H. Bai, S. Hemour, Y. P. Zhao, D. Houssameddine, K. Wu, H. Guo, and C.-M. Hu, Appl. Phys. Lett. 106, 152403 (2015).

${ }^{48}$ J. Sato, M. Oogane, H. Naganuma, and Y. Ando, Appl. Phys. Express 4, 113005 (2011). 